\section{Clinical Laboratory Reagents: The Regulatory Hurdle}

\author{
Peter A. Takes, Stereotaxis, Inc.
}

Today's clinical laboratory has a diversity of reagents and protocols from which to choose: a variety of clinical chemistry reagents, antibodies, and molecular probes, among others. These materials facilitate serum and urine analysis, histology, hematology, cytology, and a host of additional sensitive and specific assays.

Regardless of the application, labs may see an array of labeling statements indicating a reagent's general use: "For Laboratory Use Only" [LUO]; "For Research Use Only" [RUO]; "For Investigational Use Only" [IUO]; "For in Vitro Diagnostic Use" [IVD]; and "Analyte Specific Reagent" [ASR]. These preparations are often employed without realizing the course manufacturer's must follow in order to provide reagents, and the reasons for these designations.

The various labeling indications (LUO, RUO, IUO, IVD, and ASR) are defined in the U.S. Code of Federal Regulations $[\mathrm{CFR}]^{1}$, and have distinct regulatory inferences. The manufacture and supply of clinical laboratory reagents falls under the purview of the U.S. Food and Drug Administration [FDA]. When these mandatory statements are employed, an assumption is made about the reagent's background and developmental history, as well as the qualifications of the end user. ${ }^{2}$

Clinical products ("medical devices") fall into one of three classes, as defined by the Medical Device Amendments of $1976 .^{3}$ These definitions are based on the level of risk to the patient (by treatment or misdiagnosis) assumed by FDA, and are thus evaluated as to their "safety and effectiveness". Class I products are those that pose minimal potential for harm to the patient, and are subject to "general controls". Among general controls are the standard requirements for assurance of manufacturing process integrity and product traceability (current Good Manufacturing Practices, cGMP). The concept of cGMP applies to all product categorizations. Reagents grouped into Class II are those where general controls are not considered sufficient to ensure safety and effectiveness, thus making "special controls" necessary. Special controls can be items such as mandatory industry performance standards which must be met. Class III, the strictest categorization, is reserved for devices which support or sustain human life, or are considered to present a potentially immeasurable risk of illness or injury in the event of device malfunction or a misread result. Many new technologies fall, by default, into the latter classification until sufficient industry and laboratory expertise is demonstrated in the use of the reagent in question.

Once determined to fit into one of these classifications, the device's usage labeling is defined based upon its stage of development and/or the level of training necessary to acquire an accurate result. General purpose reagents, labeled "LUO", are those whose applications "are generally known by persons trained in their use", and have "general laboratory application". An example would be hydrochloric acid. These may be produced and sold by manufacturers with relatively few restrictions.

The labeling of reagents as "Research Use Only" can be misleading if one is not familiar with the regulatory requirements. FDA's interpretation of "RUO" is distinctly different than that assumed by the end user or the uninitiated manufacturer. Products so labeled are considered by FDA to be "in the labo- ratory research phase of development". Consequently, it is expected these reagents will progress to IUO and subsequent IVD status, a progression which requires the acquisition of a significant amount of clinical research data. For Class II or Class III products, this can be considerable. Many Class I products are now exempt from FDA review prior to commercialization. Manufacturers have historically employed RUO designations for non-diagnostic materials sold to research labs. Thus, to avoid in part what FDA might consider "misuse" of RUO labeling, many manufacturers now require end users to complete and return statements demonstrating awareness of the reagent's RUO status (certification programs). This would be required if end users wish to make subsequent purchases of the same product.

The investigational use, or "IUO", statement indicates that a reagent is under clinical study, and largely may be shipped or delivered only to those institutions involved in data acquisition for the manufacturer. Distribution of IUO reagents outside this scope is prohibited.

The "IVD" designation is reserved for reagents which have received FDA clearance or approval for marketing. Generally, this is the result of lengthy applications made to FDA for clearance/ approval, supported by extensive technical, manufacturing, regulatory compliance, and clinical data.

The ASRs present the newest labeling alternative., ${ }^{4,5}$ The FDA defines ASRs as "...antibodies, both polyclonal and monoclonal, specific receptor proteins, ligands, nucleic acid sequences, and similar reagents which, through specific binding or chemical reaction with substances in a specimen, are intended for use in a diagnostic application for identification and quantification of an individual chemical substance or ligand in biological specimens." Although most ASR designations will allow reagents to become available to the end user more quickly (FDA considers most of these to be of relatively low risk to public health), there are certain restrictions for companies indicating their products to be ASRs. There should be no relative impact on academic or research labs that do not perform clinical testing. However, since ASRs are presumed to be defined by their lack of established analytical and performance characteristics, the dissemination of information (even to research labs) on applicable methods and techniques is restricted. This includes possible assistance in test optimization. Further, the direct effect on clinical laboratories using ASRs may extensive, including the need to open their doors to FDA inspection, an area previously clearly outside of the Agency's purview.

As can be noted, the considerations of manufacturers in providing research and/or clinical reagents are broad. These must include review of possible end use of products and the regulatory consequences of perceived mislabeling. The research and development leading to a reagent's creation and subsequent production scale-up are just small pieces of the puzzle. The ramifications of not using the proper regulatory designations on a product's label can be both costly and devastating, from product "recall", to market withdrawal, to fines. Diligence toward awareness of these concerns and requirements are beneficial to both the manufacturer and end user laboratory.

1) 21 CFR 809.10 .

2) Takes, P.A. 1998. What's in a label? Clin Lab News 24(4):10.

3) Takes, P.A. and L. Chambers. 1995. In Vitro Diagnostics: The FDA regulatory process. Clin Lab News 21(7):43-44.

4) Federal Register 62:62243-62260, 1997.

5) Takes, P.A. and G. Murray. 1998. The Analyte Specific Reagent rule - a new set of regulations for labs, manufacturers. Clin Lab News 24(4): 10. 


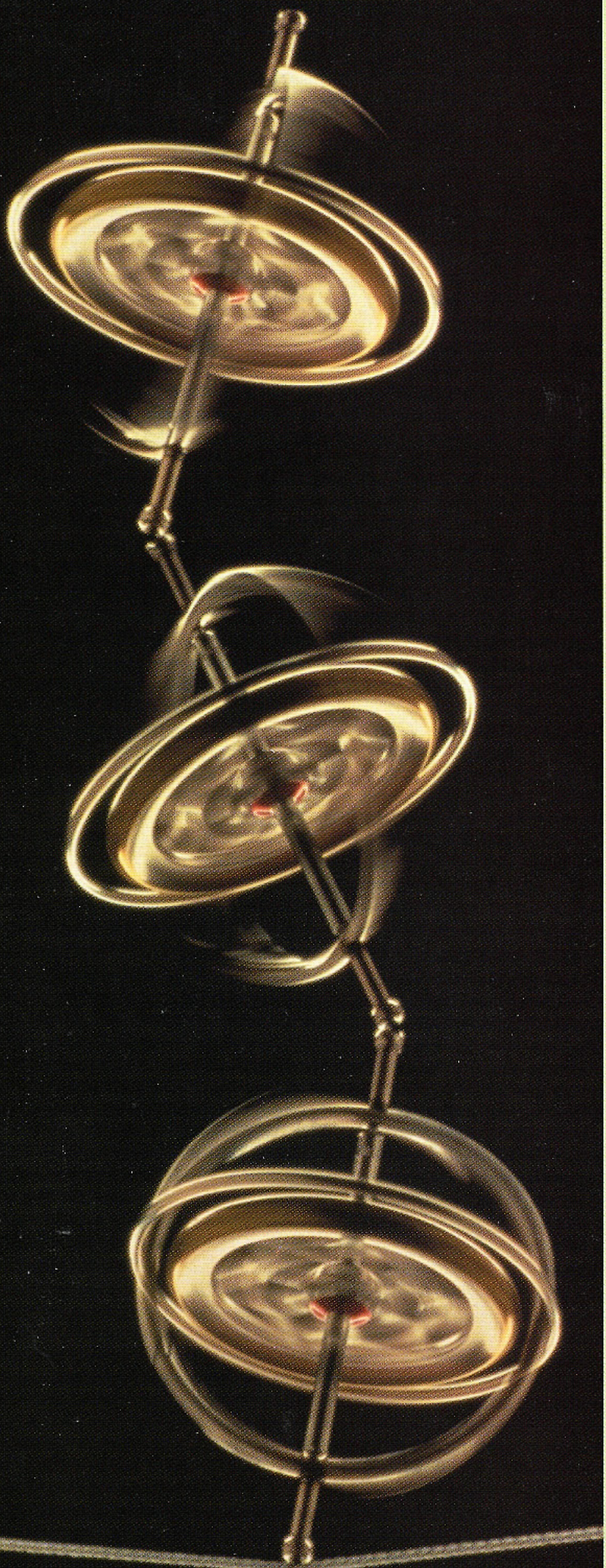

\section{Getting All Your Detectors Working Together Can Be A Tough Balancing Act. \\ That's Where Emispec Comes In.}

If balancing all components of your detectors has your head spinning, you should be talking to us.

You see, at Emispec Systems, Inc., we approach data acquisition differently. Instead of creating systems targeting one detector, we focus on integration. This concept can be applied equally to new and existing electron microscope installations. Core acquisition capabilities of our products include:

- Digital scanning for STEM.

- Digital EDX acquisition and analysis.

- EELS acquisition and analysis.

- $\mathrm{CCD}$ and TV imaging.

Integrated microscope control, imaging and spectroscopy allows automation of demanding experiments, such as spectrum imaging. Emispec enhances these capabilities with extensive on-line and off-line processing.

To find out how Emispec can help your lab keep in balance, visit our Web site today at www.emispec.com. See why we are fast becoming the leader in microscope detector technology solutions.

\section{Emispec}

Emispec Systems, Inc

2409 South Rural Road, Suite D

Tempe, Arizona 85282 USA

Phone: 480.894 .6443 - Fax: 480.894 .6458

Web: wmw.emispec.com

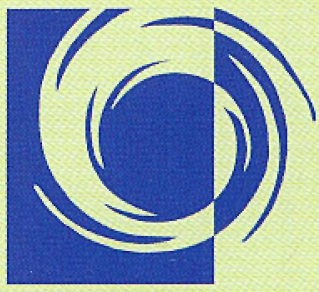

thinking beyond the box ${ }^{T M}$ 